\title{
Metodologia para análise de resultados em lipoaspiração
}

\section{Methodology for analysis of results in liposuction}

\author{
Ataliba Ronan Horta de \\ Almeida $^{1}$ \\ Andre Villani Correa \\ MAFRA $^{2}$ \\ Gnana Keith Marques de \\ Araújo $^{3}$
}

Trabalho realizado no Hospital Mater Dei, Belo Horizonte, MG,

Brasil.

Artigo submetido pelo SGP (Sistema de Gestão de Publicações)

da RBCP.

Artigo recebido: 21/10/2008 Artigo aceito: 9/4/2011

\begin{abstract}
RESUMO
Introdução: A lipoaspiração é hoje método padrão para tratamento das lipodistrofias. Entretanto, a amplitude das áreas a serem tratadas torna complexa a avaliação dos resultados perioperatórios. Método: Neste artigo, descrevemos nova tática que objetiva o controle perioperatório da lipoaspiração. Medidas pré-operatórias da espessura da prega cutânea foram realizadas com uso de adipômetro e régua. As regiões aferidas foram padronizadas. A prega axilar anterior entre a nona e décima costela é utilizada como referência para o resultado da lipoaspiração abdominal superior, inferior bilateral e de flancos. Assimetrias ou hipocorreção das áreas tratadas são minimizadas. Durante a consulta médica há a demonstração com números das medidas no pré e pós-operatório. Conclusão: Esta conduta evita análises subjetivas. Deste modo a relação médico-paciente é facilitada com a redução do risco de conflito.
\end{abstract}

Descritores: Lipectomia. Cirurgia Plástica. Estética. Tecido Adiposo.

\begin{abstract}
Introduction: Liposuction is the standard method for lipodistrophy treatment. Perioperative results evaluation is difficult due to amplitude of areas to be treated. Methods: This article describes a new method of perioperative results control during liposuction. Cutaneous fold thickness of standard areas are measured using adipometer and scale in the preoperative period. Cutaneous thickness on anterior axilar fold between ninth and tenth ribs is used as reference for superior and inferior abdominal and flanks liposuction. Treated areas asymmetries or hipocorrection are minimized. During medical consulting there is a demonstration of preoperative and postoperative measured values. Conclusion: This conduct avoids subjective analysis facilitating doctor patient relation with conflict risk reducing.
\end{abstract}

Keywords: Lipectomy. Plastic Surgery. Esthetics. Adipose Tissue.

1. Médico Cirurgião Plástico. Preceptor do Centro de Formação e Treinamento em Cirurgia Plastica do Hospital Mater Dei. Membro Titular da Sociedade Brasileira de Cirurgia Plástica. Membro do Corpo Clínico do Hospital Mater Dei, Belo Horizonte, MG, Brasil.

2. Médico Cirurgião Plástico. Membro Especialista da Sociedade Brasileira de Cirurgia Plástica. Membro do Corpo Clínico do Hospital Mater Dei, Belo Horizonte, MG, Brasil.

3. Mestre em Cirurgia pela Faculdade de Medicina da UFMG. Médica Cirurgiã Plástica. Membro Titular da Sociedade Brasileira de Cirurgia Plástica. Membro do Corpo Clínico do Hospital Mater Dei, Belo Horizonte, MG, Brasil. 


\section{INTRODUÇÃO}

A lipoaspiração foi descrita por Gerard Illouz, em 1977, com o objetivo de tratar as lipodistrofias ${ }^{1,2}$. A lipoaspiração foi integrada ao arsenal terapêutico do cirurgião plástico por meio de estudos e prática cirúrgica. Trabalhos científicos certificaram as indicações, limitações e complicações desta técnica, tornando-a segura para uso ${ }^{2-7}$.

Entretanto, a amplitude das áreas a serem tratadas muitas vezes dificulta a análise dos resultados perioperatórios ${ }^{8}$. Transformar em números essa avaliação não acaba com as interpretações pessoais por parte dos cirurgiões e dos pacientes, mas permite demonstração menos subjetiva.

O tecido subcutâneo é dividido em camada areolar, superficial e camada lamelar ou reticular, profunda. A camada lamelar é mais susceptível ao aumento de sua espessura nos casos de acúmulo de adiposidade. $\mathrm{O}$ número de células adiposas presentes na camada lamelar é o principal responsável pela hipertrofia e aumento da espessura do panículo adiposo, podendo uma célula adiposa vir a ter até cem vezes o seu volume original ${ }^{9,10}$. O tecido celular subcutâneo apresenta comportamento diferente em determinadas áreas corporais. Regiões corporais onde a pele é mais espessa e firme, por exemplo, no tórax, apresentam maior desenvolvimento da camada areolar em detrimento da lamelar ${ }^{9,10}$

Neste artigo, descrevemos tática que objetiva o maior controle perioperatório da lipoaspiração, associado à diminuição de queixas e complicações pós-operatórias relacionadas às possíveis assimetrias e hipocorreções das áreas tratadas.

\section{MÉTODO}

Foram avaliadas doze pacientes submetidas a tratamento de lipodistrofia através da lipoaspiração, cirurgias estas realizadas pelos autores. Com o auxílio de adipômetro e a utilização de régua, foram realizadas medidas da espessura da prega cutânea na linha axilar média entre a nona e a décima costelas. Esta medida foi considerada o padrão e tomada como referência para aquela paciente. As áreas a serem tratadas foram medidas com a mesma metodologia.

As referências anatômicas para mensuração das áreas de lipoaspiração são a linha alba acima da cicatriz umbilical, para o abdome superior, a linha imaginária sobre os músculos reto abdominais, para abdome inferior, as cristas ilíacas e seus tubérculos, para flancos, e a área infraescapular perpendicular às fibras do músculo grande dorsal, para o dorso.

As medidas foram anotadas no prontuário médico $\mathrm{e}$ as pacientes foram fotografadas (Figura 1). A paciente foi submetida à marcação da área a ser aspirada no pré-operatório e encaminhada para cirurgia. Foi utilizada a técnica de lipoaspiração úmida ou infiltrativa, com a infiltração de solução de cloreto de sódio a $0,9 \%$ e adrenalina na proporção de $1 / 500.000$, sob anestesia peridural associada à sedação venosa, realizadas pelo mesmo anestesiologista.

Durante o procedimento cirúrgico, as áreas tratadas foram repetidamente medidas para aproximar por lipoaspiração as dimensões de suas pregas cutâneas àquelas adquiridas na região de referência (Figuras 2 a 4). O término da cirurgia foi determinado pela maior proximidade dos valores.

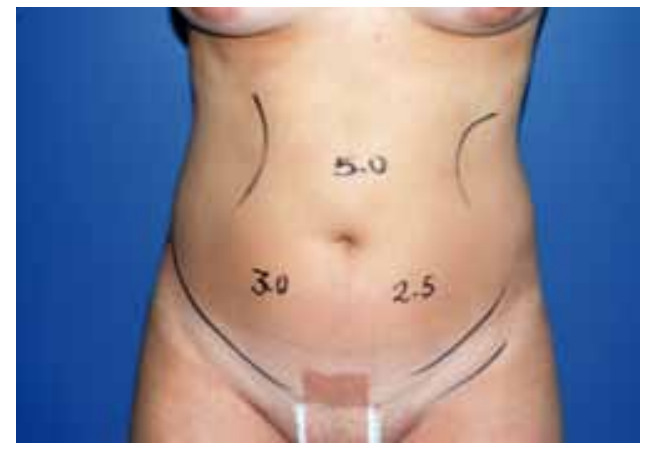

Figura 1 - Áreas de tratamento no abdome, medidas no pré-operatório.

Estas medidas foram repetidas em avaliações pós-operatórias até a alta médica. Seis meses após a cirurgia, quando aproximadamente $80 \%$ do edema já apresentava regressão, as pacientes foram novamente submetidas a mensuração das pregas cutâneas e os valores foram comparados aos dados pré-operatórios (Figuras 3 a 8).

Abaixo exemplificamos, por meio de um caso clínico, a condução de uma paciente conforme as orientações deste trabalho descrito. As Figuras 8 e 9 são de pré-operatório. Em um primeiro momento, com o espoliar do pós-operatório imediato, a paciente emagrece, e ainda há um edema residual na área operada (Figuras 10 e 11). Pode-se observar que o valor de referência dado pela medida na linha axilar anterior entre a $9^{\mathrm{a}}$ e $10^{\mathrm{a}}$ costelas se reduz de $1,2 \mathrm{~cm}$ para $0,7 \mathrm{~cm}$. Observa-se, também, a homogeneidade dos valores adquiridos nas áreas tratadas. Com o passar do tempo e o equilíbrio corporal, este valor de referência retorna para $1,2 \mathrm{~cm}$ - paciente em consulta de pós-operatório de 22 meses (Figura 12). Vale ressaltar que não houve alteração de peso corporal nesta paciente.

\section{RESULTADOS}

As Figuras 3 a 15 ilustram alguns casos dessa casuística.

A Tabela 1 apresenta comparação entre as medidas padrão e os valores médios das pregas cutâneas analisadas no estudo de lipoaspiração, para cada paciente, também apresentadas nas Figuras 16 e 17.

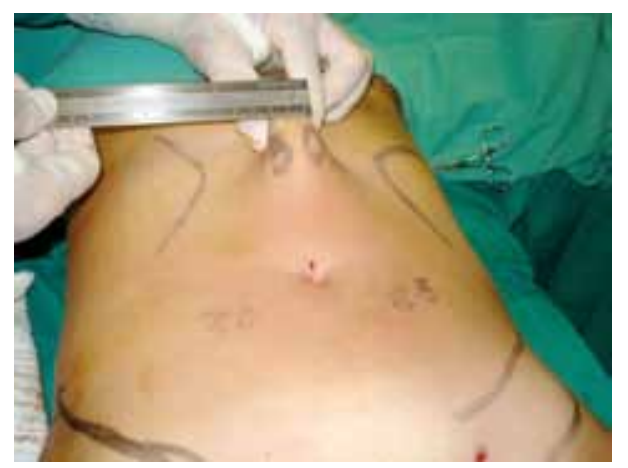

Figura 2-Mensuração pré-operatória da espessura da prega cutânea no flanco esquerdo com um adipômetro. 

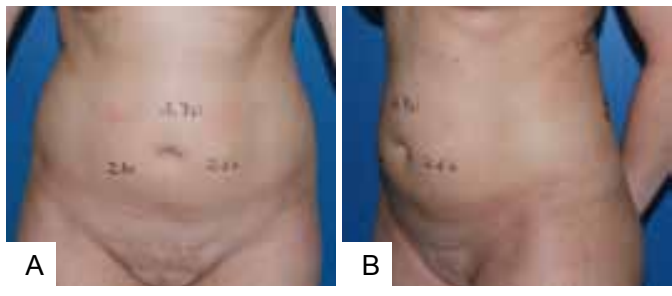

Figura 3 - Areas de tratamento no abdome. Medidas de prega cutânea no pré-operatório. Posição de frente (vista anterior). B. Áreas de tratamento no abdome. Medidas de prega cutânea no préoperatório (vista obliqua).
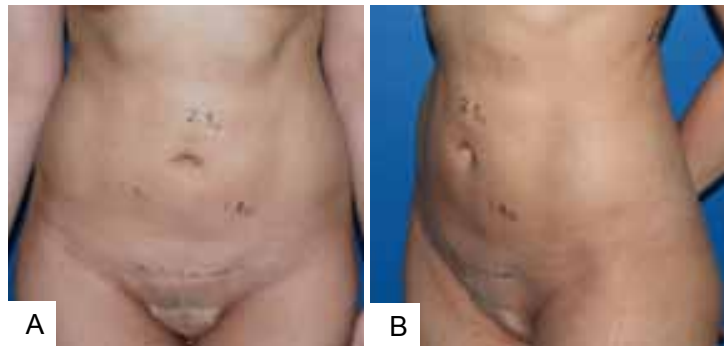

Figura 4 - Áreas de tratamento no abdome. Medidas de prega cutânea no pós-operatório. Posição de frente (vista anterior). B: Áreas de tratamento no abdome. Medidas de prega cutânea no pós-operatório (vista oblíqua).
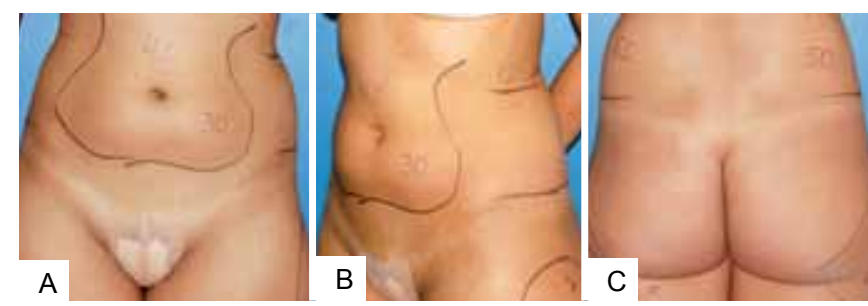

Figura 5-A: Áreas de tratamento no abdome. Medidas de prega cutânea no pré-operatório. Posição de frente (vista anterior). B: Áreas de tratamento no abdome. Medidas de prega cutânea no pré-operatório (vista oblíqua). C: Áreas de tratamento no abdome. Medidas de prega cutânea no pré-operatório (vista posterior).
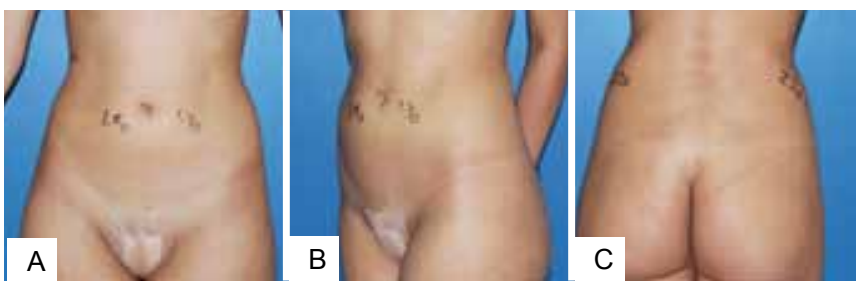

Figura 6-A: Áreas de tratamento no abdome. Medidas de prega cutânea no pós-operatório. Posição de frente (vista anterior). B: Áreas de tratamento no abdome. Medidas de prega cutânea no pós-operatório (vista oblíqua). C: Áreas de tratamento no abdome. Medidas de prega cutânea no pós-operatório (vista posterior).
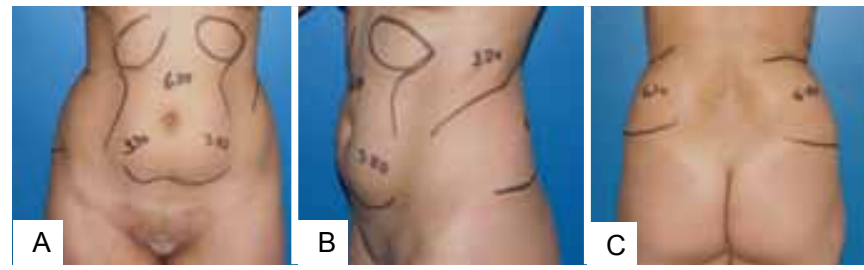

Figura 7 -A: Areas de tratamento no abdome. Medidas de prega cutânea no pré-operatório. Posição de frente (vista anterior). B: Areas de tratamento no abdome. Medidas de prega cutânea no pré-operatório (vista oblíqua). C: Áreas de tratamento no abdome. Medidas de prega cutânea no préoperatório (vista posterior).
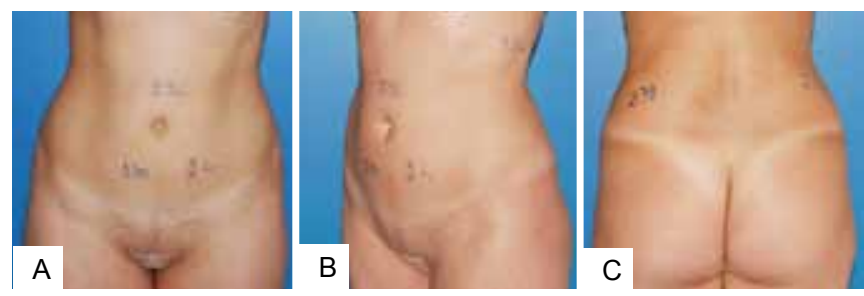

Figura 8-A: Áreas de tratamento no abdome. Medidas de prega cutânea no pós-operatório. Posição de frente (vista anterior). B: Áreas de tratamento no abdome. Medidas de prega cutânea no pós-operatório (vista oblíqua). C: Áreas de tratamento no abdome. Medidas de prega cutânea no pós-operatório (vista posterior).
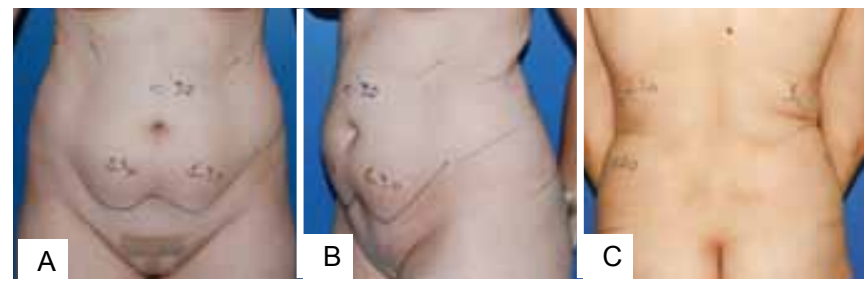

Figura 9-A: Áreas de tratamento no abdome. Medidas de prega cutânea no pré-operatório. Posição de frente (vista anterior). B: Áreas de tratamento no abdome. Medidas de prega cutânea no pré-operatório (vista oblíqua). C: Áreas de tratamento no abdome. Medidas de prega cutânea no pré-operatório (vista posterior).
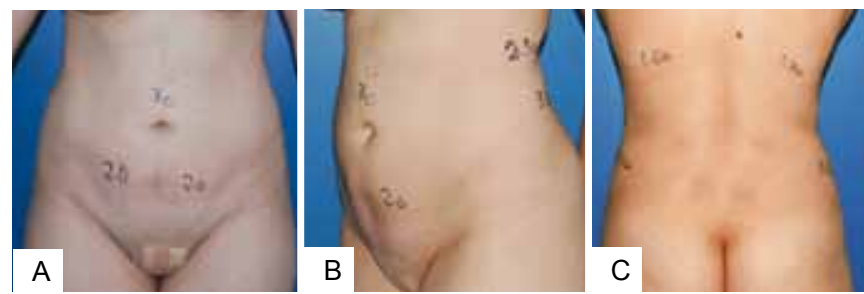

Figura 10-A: Áreas de tratamento no abdome. Medidas de prega cutânea no pós-operatório. Posição de frente (vista anterior). B: Areas de tratamento no abdome. Medidas de prega cutânea no pós-operatório (vista oblíqua). C: Áreas de tratamento no abdome. Medidas de prega cutânea no pós-operatório (vista posterior). 


\section{DISCUSSÃO}

As assimetrias pré-operatórias do contorno corporal são demonstradas numericamente para o paciente.

A prega cutânea na linha axilar média entre a $8^{\mathrm{a}} \mathrm{e} 9^{\mathrm{a}}$ costelas foi padronizada como medida padrão porque é uma área corporal que se altera pouco com as oscilações de peso do indivíduo e não é uma área que lipoaspiramos de rotina na nossa prática clínica. Desta forma, no controle pré, per e pós-operatório, esta área torna-se uma referência para compararmos as alterações corporais do indivíduo.

Pode-se observar que, no pré-operatório, as medidas das pregas cutâneas de cada paciente tendiam a heterogeneidade (Tabela 1). Após o tratamento por lipoaspiração, estas medidas tornaram-se homogêneas e uniformes, com aproximação à medida padrão (Tabela 1).

Ao observarmos as tabelas e gráficos, podemos ter a comprovação numérica: o cálculo do desvio padrão das

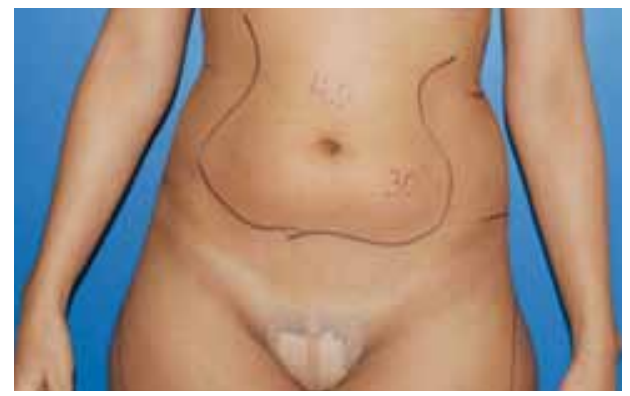

Figura 11 - Pré-operatório, vista anterior.

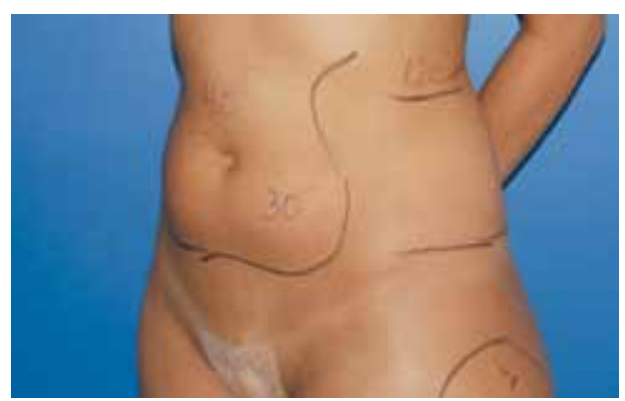

Figura 12 - Pré-operatório, vista oblíqua.

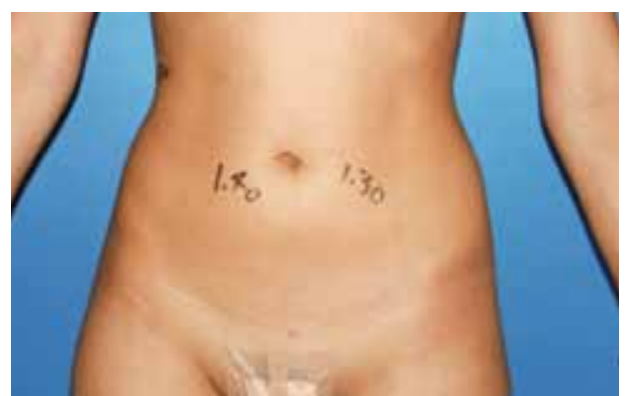

Figura 13 - Pós-operatório de 3 meses, vista anterior.

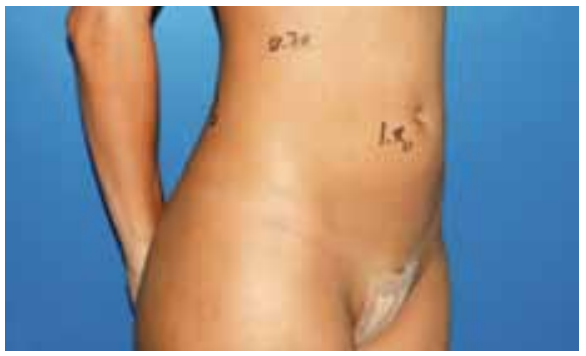

Figura 14 - Pós-operatório de 3 meses, vista oblíqua.

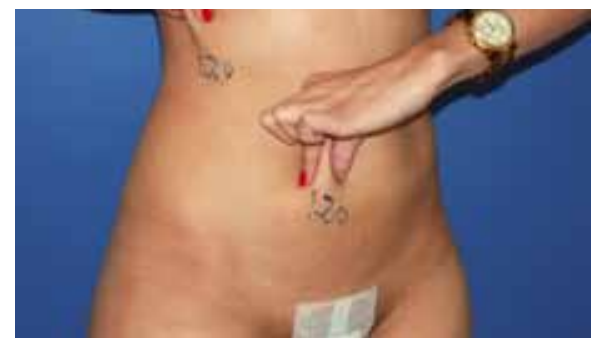

Figura 15 - Pós-operatório de 22 meses, vista anterior, ilustrando a manobra bidigital feita pela própria paciente.

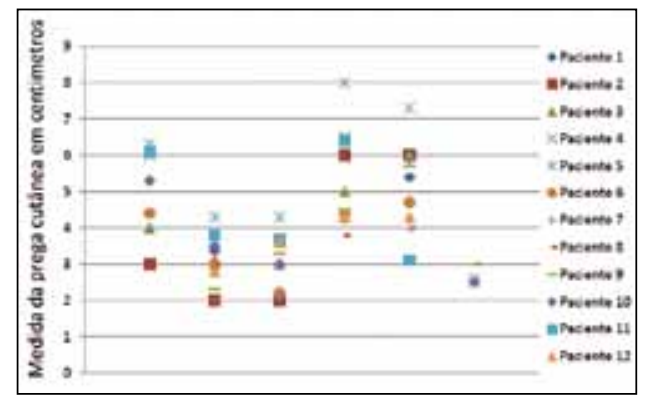

Figura 16 - Medida das pregas cutâneas no pré-operatório.

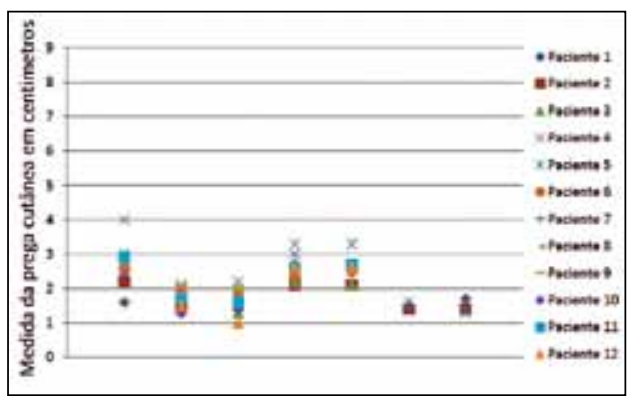

Figura 17-Medida das pregas cutâneas no pós-operatório.

medidas do pré-operatório é mais heterogêneo (valor maior), se comparados com o cálculo deste valor no pós-operatório destas mesmas medidas, nas mesmas pacientes do estudo. No pós-operatório, o desvio padrão é um valor menor. Isto evidencia a homogeneidade dos valores de medidas para aquela paciente, ou seja, resultado homogêneo do procedimento cirúrgico. 
Tabela 1 - Comparação entre as medidas padrão e os valores médios em centímetros das pregas cutâneas analisadas no estudo de lipoaspiração, para cada paciente.

\begin{tabular}{|c|c|c|c|c|c|c|}
\hline \multirow[t]{2}{*}{ Paciente } & \multicolumn{3}{|c|}{ Pré-operatório } & \multicolumn{3}{|c|}{ Pós-operatório (6 meses) } \\
\hline & Padrão ${ }^{1}$ & Média $^{2}$ & DesvPad $^{3}$ & Padrão ${ }^{1}$ & Média $^{2}$ & DesvPad $^{3}$ \\
\hline & $(\mathbf{c m})$ & $(\mathbf{c m})$ & & $(\mathbf{c m})$ & $(\mathbf{c m})$ & \\
\hline 1 & 1,0 & 3,75 & 1,66 & 1,4 & 1,95 & 0,77 \\
\hline 2 & 1,7 & 3,45 & 2,02 & 1,2 & 1,77 & 0,42 \\
\hline 3 & 1,2 & 3,70 & 1,69 & 0,7 & 1,54 & 0,62 \\
\hline 4 & 1,3 & 4,42 & 2,02 & $\mathrm{X}$ & 2,02 & 0,79 \\
\hline 5 & 2,0 & 5,36 & 2,25 & 2,3 & 2,65 & 0,62 \\
\hline 6 & 0,9 & 3,27 & 1,51 & $\mathrm{X}$ & 2,26 & 0,34 \\
\hline 7 & 1,1 & 2,9 & 1,33 & 1,9 & 2,1 & 0,63 \\
\hline 8 & 1,5 & 3,16 & 1,00 & 1,6 & 2,22 & 0,50 \\
\hline 9 & 2,0 & 3,56 & 1,54 & 2,0 & 2,28 & 0,34 \\
\hline 10 & 2,3 & 3,56 & 1,28 & 1,5 & 1,87 & 0,65 \\
\hline 11 & 1,2 & 4,05 & 1,94 & 1,1 & 2,1 & 0,73 \\
\hline 12 & 1,0 & 3,2 & 1,4 & 1,0 & 1,74 & 0,8 \\
\hline Média/DesvPad & & 3,69 & 0,66 & & 2,04 & 0,29 \\
\hline
\end{tabular}

1. Padrão - Medido, em centímetros, da prega cutânea correspondente à linha médio-axilar, entre a $9^{\mathrm{a}}$ e $10^{\mathrm{a}}$ costelas. 2 . Média - Média aritmética dentre todas as medidas, em centímetros, de cada paciente, sendo elas: Abdome Superior - linha alba supraumbilical; Abdome Inferior - linha imaginária sobre o músculo reto abdominal à direita e à esquerda; Flanco - ponto entre a crista ilíaca e tubérculo ilíaco à direita e à esquerda; Dorso - área infraescapular perpendicular ao músculo grande dorsal à direita e à esquerda. 3. DesvPad - Desvio Padrão é um valor que, neste estudo, foi calculado através do programa Excel (Office) e informa se a população - ou as características - em estudo é homogênea quanto aos dados estudados. Quanto maior o valor calculado, mais heterogênea é a população (ou os valores) estudada, sendo o inverso totalmente verdadeiro e absoluto quando atinge valores menores que 0,05. Este cálculo estima o Desvio Padrão baseado em uma amostra de dados. O Desvio Padrão é a medida que demonstra o quão os valores estão dispersos do valor médio da amostra. Esta função assume que os dados em estudo são uma amostra da população. O Desvio Padrão é calculado utilizando-se do método de "n-1". Esta função utiliza a seguinte fórmula: $\sqrt{\sum(x-\bar{x})^{2}}$ Enquanto x é a média da amostra de dados estudados (valor1, valor2, etc.) e n é o tamanho da amostra.

Ilouz ${ }^{5}$ relatou que complicações em lipoaspiração são divididas em dois grupos. O primeiro está relacionado às alterações e complicações cirúrgicas e sistêmicas e continuam mínimas na técnica tradicional. O segundo grupo, quando se ultrapassam os limites de segurança, se relaciona aos resultados desfavoráveis e às sequelas, as quais são numerosas e complexas ${ }^{5}$. Pacientes esperam da cirurgia plástica os melhores resultados possíveis ${ }^{11}$. Por meio de avaliações subjetivas, impressões conflitantes entre cirurgião e paciente podem surgir. Com o uso de um método da avaliação quantitativa para a análise da lipodistrofia de cada paciente, a demonstração objetiva dos resultados cirúrgicos é possível.

Anova tática de metodologia numérica para análise de resultados em lipoaspiração pode ser utilizada durante cirurgia para a obtenção de uma referência de espessura para as pregas cutâneas abdominal, de dorso e de flancos após a lipoaspiração. Outra vantagem do método aqui descrito é a facilidade em demonstrar a ocorrência eventual de ganho ponderal. Isto é demonstrado pela comparação das medidas no pós-operatório imediato e tardio em relação à área considerada medida padrão de referência para aquela paciente (linha média axilar, $9^{\mathrm{a}}$ costela).

Nós temos utilizado esta tática em nosso serviço nos últimos dois anos, com fácil execução, baixo custo e resultados satisfatórios. A nova metodologia traz mais segurança ao cirurgião plástico, associado à redução nos conflitos e melhora na relação médico-paciente.

\section{REFERÊNCIAS}

1. Souza Pinto EB, Abdalla PCSP, Maciel CM. História da lipoaspiração. In: Carrerão S, Cardim V, Goldenberg D, eds. Cirurgia Plástica. São Paulo: Atheneu;2005. cap.6.

2. Toledo LS, Giovannetti M. Lipoaspiração e lipoenxertia nas deformidades do abdômen, dorso e flancos. In: Mélega JM, ed. Cirurgia Plástica: fundamentos e arte - cirurgia estética. Rio de Janeiro: Medsi;2003. p.629-38.

3. Coleman WP 3rd. Powered liposuction. Dermatol Surg. 2000; 26(4):315-8

4. Pollack SV. Liposuction of the abdomen. The basics. Dermatol Clin. 1999;17(4):823-34.

5. Illouz YG. Complications of liposuction. Clin Plast Surg. 2006;33(1): 129-63.

6. Illouz YG. Lipesculpture et chirurgie de la silhouette. Chirurgie plastique reconstructrice et esthétique. Encycl Med Chir Edidions Scientifiques et Médicales. Paris:Elsevier;1998. p.45-120.

7. Hunstad JP, Aitken ME. Liposuction: techniques and guidelines. Clin Plast Surg. 2006;33(1):13-25.

8. Chang KN. The use of intraoperative grid pattern markings in lipoplasty. Plast Reconstr Surg. 2004;114(5):1292-7.

9. Avelar J, Illouz YG. Anatomia cirúrgica e distribuição do tecido celular no organismo humano. In: Lipoaspiração. Rio de Janeiro:Hipocrates;1986. p.45-57.

10. Duarte AC, Castellani FR. Medidas antropométricas. In: Semiologia nutricional. São Paulo:Axcel Books do Brasil;2002. p.34-57.

11. Augustin M,Zschocke I, Sommer B, Sattler G. Sociodemographic profile and satisfaction with treatment of patients undergoing liposuction in tumescent local anesthesia. Dermatol Surg. 1999;25(6):480-3.

\section{Correspondência para:}

Ataliba Ronan Horta de Almeida

Rua Gonçalves Dias, 1181 - $1^{\circ}$ andar - Belo Horizonte MG, Brasil - CEP: 30140-091

E-mail: ronan@ronanhorta.com.br 